This document is the Accepted Manuscript version of a Published Work that appeared in final form in Journal of Physical Chemistry B, copyright (C) American Chemical Society after peer review and technical editing by the publisher. To access the final edited and published work see http://pubs.acs.org/doi/abs/10.1021/acs.jpcb.5b08110.

\title{
Molecular Dynamics Simulations of the Interaction between Water Molecules and Aggregates of Acetic or Propionic Acid Molecules
}

\author{
Bastien Radola, ${ }^{1}$ Sylvain Picaud, ${ }^{1 *}$ Delphine Vardanega, ${ }^{1,2^{*}}$ \\ Pál Jedlovszky ${ }^{3,4,5}$
}
${ }^{1}$ Institut UTINAM - UMR 6213, CNRS, Univ. Bourgogne Franche-Comté, F-25000 Besançon Cedex, France

${ }^{2}$ PhLAM - UMR8523, CNRS, Univ. Lille 1, F-59655 Villeneuve d'Ascq, France

${ }^{3}$ Laboratory of Interfaces and Nanosized Systems, Institute of Chemistry, Eötvös

Loránd University, Pázmány Péter stny, 1/a, H-1117 Budapest, Hungary

${ }^{4}$ HAS Research Group of Technical Analytical Chemistry, Szt. Gellért tér 4, H-1111 Budapest, Hungary

${ }^{5}$ EKF Department of Chemistry, Eszterházy tér 1, H-3300 Eger, Hungary

Running title: interaction of water molecules with carboxylic acid aggregates

*Electronic mail: sylvain.picaud@univ-fcomte.fr (S.P.) 


\section{Abstract:}

Water adsorption around small acetic and propionic acid aggregates has been studied by means of molecular dynamics simulation in the temperature range of 100-250 K as a function of the water content. Calculations have shown that acetic and propionic acid molecules behave similarly, and that both the temperature and the water content have a strong influence on the behavior of the corresponding systems.

Two situations have been evidenced for the acid-water aggregates, corresponding either to water adsorption on large acid grains at very low temperatures, or to the formation of droplets consisting of acid molecules adsorbed at the surface of water aggregates at higher temperatures and high water content. At low water content and high temperature, only a partial mixing between water and acid molecules has been observed.

The results of the present simulations emphasize the need for further experimental and simulation works to achieve a better characterization of the effects of both temperature and humidity on the behavior of organic aerosols in the Troposphere. 


\section{Introduction}

Carbonaceous aerosols constitute a significant subgroup of atmospheric aerosols that consists of elemental carbon (black carbon, freshly emitted soot), organic carbon, and, often, a mixture of both elemental and organic carbon (brown carbon).[1] These aerosols can be primarily emitted, or secondarily formed in the atmosphere, for instance, as oxidation products of gaseous organic precursors with low vapor pressure.[2] However, due to the complexity of their chemical nature, the significance of carbonaceous aerosols in driving physical and chemical atmospheric processes is still very poorly understood.

Carbonaceous aerosols play an important role in climatic processes, having both direct and indirect effects.[3-5] Direct effects on climate come from scattering and absorbing solar and terrestrial radiations, whereas indirect effects are directly connected to the ability of these particles to act as cloud condensation $(\mathrm{CCN})$ and ice formation (IN) nuclei.[6] CCN are particles that in the presence of supersaturated water vapour initiate condensation and become centers of cloud or fog droplets, whereas IN are the necessary ingredients for heterogeneous nucleation processes leading to the formation of ice particles.[7] Thus, understanding the mechanisms that drive the interaction between carbonaceous aerosols and surrounding water molecules it is of fundamental interest to better assess the role that these $\mathrm{CCN}$ and IN play in atmospheric processes.

In addition, as chemical characterization of these aerosols has revealed that organic compounds are the major constituents of fine aerosols, [7] it is now clear that water condensation and heterogeneous ice nucleation on organic species cannot be ignored.[8]

Among these organic compounds, carboxylic acids are one of the most abundant species. Indeed, these molecules are emitted directly to the atmosphere via several biogenic (biomass burning) and anthropogenic (pharmaceutical and chemical industries, biofuels) sources, and they can also be formed secondarily, via the photo-oxidation of hydrocarbons in both gas and aqueous phases of clouds. [9] Focusing on the interaction between carboxylic acids and water is thus very important in the context of tropospheric physico-chemistry, and combination of field measurements, laboratory experiments and modeling studies appears mandatory to achieve a better characterization of the corresponding systems.

Because of the high variability of the organic aerosol phase in terms of sources, composition and evolution, the modeling of ideal systems by computer simulations appears to be appealing. Such modelling can be the first step towards better understanding aerosol behavior, 
using information at the molecular scale. More specifically, interactions between the organic phase and the surrounding water molecules can be investigated in detail on the basis of a rigorous description of the corresponding interactions. However, even simplified, a realistic modeling of these systems has to consist of, at least, hundreds of molecules. As a consequence, quantum calculations will certainly not be feasible, instead, long, classical approaches based on a simplified, but nonetheless relevant description of the organic aerosol - water interactions should be used.

For instance, classical interaction models have been shown to correctly reproduce experimental results when characterizing the interaction between small volatile organic compounds and ice surfaces using either molecular dynamics simulations [10-13] or Monte Carlo calculations [14-23]. Then, classical approaches have been used to characterize the interaction between large organic aggregates and water molecules, as a function of the temperature and of the water content in the corresponding simulations.[24-26] Similarly, a few molecular dynamics simulation studies have been recently devoted to the characterization of the reverse situation, i.e., when big water droplets are coated by various organic molecules [27-32]. These two approaches, namely water droplets interacting with organic matter, and organic aggregates interacting with water, led to similar conclusions, in particular, regarding the state of mixture of the aerosol particles. Indeed, water and organic molecules have been found to form either mixed or demixed phases depending on the temperature, the water:acid ratio, and, more importantly, on the type of the organic molecules. Thus, using the $\mathrm{O}: \mathrm{C}$ ratio as a proxy for characterizing the organic aerosol hydrophilicity, as recently suggested by Shill and Tolbert, [8] could not be an oversimplification. In contrast, a systematic study of a large series of carboxylic acid molecules should be undertaken before any global conclusion (if any) can be drawn.

As a consequence, we complement our previous works on oxalic [24], malonic [25] and formic [26] acids by considering here the case of aggregates formed by acetic and propionic acid molecules. Indeed, after characterizing the influence of the internal conformation of the acid molecule (difference between oxalic and malonic acids), and the influence of the chemical type of acid (mono- vs. di-carboxylic acid), the present study aims at investigating the effect of the length of the hydrophobic tail that could also play a significant role in the interaction between the acid and water molecules. 
The computational details of our simulation study are given In Section 2, whereas the corresponding results are detailed in Section 3. These results are then discussed in Section 4 and the main conclusions of the present study are summarized in Section 5.

\section{Computational Details}

Molecular dynamics (MD) simulations of acetic $\left(\mathrm{CH}_{3} \mathrm{COOH}\right)$ and propionic acid $\left(\mathrm{CH}_{3} \mathrm{CH}_{2} \mathrm{COOH}\right)$ aggregates interacting with water molecules have been carried out for different compositions using the GROMACS open-source program package.[33] The potential energy of the systems investigated has been calculated as the sum of the atom-atom pairwise interaction energies between the interacting species. These interaction energies have been represented by combination of Lennard-Jones (6-12) and Coulombic terms, whose parameters for the different atoms are given in Table 1. Lennard-Jones parameters and partial charges for the acid molecules were taken from the OPLS-AA library,[34,35] and their geometries were determined using the Automated Topology Builder (ATP) tool.[36-38]

In order to be consistent with our previous studies,[24-26] water molecules have been represented by the five-site TIP5P model. [39] These molecules have been kept rigid in the simulations, whereas no constraint has been applied to acid molecules. The Lennard-Jones interactions have been cut beyond $1.4 \mathrm{~nm}$, and the Particle Mesh Ewald (PME) method has been used to take into account the long-range electrostatic interactions. The geometry of the acid and water molecules is shown in Fig. 1.

Simulations have been performed in the $(N, V, T)$ ensemble with a timestep of $0.1 \mathrm{fs}$. The temperature has been controlled by means of the Berendsen thermostat during equilibration, and using the Nose-Hoover algorithm for production runs. Indeed, although the Berendsen method allows quick convergence of the temperature value, which is of great interest for stabilization runs, it doesn't yield a correct thermodynamical ensemble. In contrast, the Nose-Hoover thermostat converges more slowly but corresponds to simulations in the correct thermodynamic ensemble.

First, MD simulations of neat acid aggregates have been performed, by randomly placing 120 acid molecules in a cubic simulation box with periodic boundary conditions; this cubic box had an edge length of $6 \mathrm{~nm}$ in the case of acetic, and $7 \mathrm{~nm}$ in the case of propionic acid. The 
system was then equilibrated at $100 \mathrm{~K}$ during $1 \mathrm{~ns}$, which was sufficiently long to obtain the formation of a stable, large and compact aggregate composed of the 120 acid molecules, being completely isolated from its periodic images. Further simulations with larger numbers of acid molecules in the box showed the same clustering behavior, at least until 240 molecules (the maximum of molecules that was considered here), as already observed for formic and malonic acids.[25,26] We have thus chosen to work with aggregates made of 120 acid molecules, which appeared as a good compromise between the relevance and the time cost of the simulations performed with large number of additional water molecules.

Indeed, to simulate the effect of increasing humidity on the behavior of the acid aggregates, five different systems in the case of acetic, and four in the case of propionic acid have been created by adding different amount of water molecules, being randomly scattered in the neighborhood of the stabilized acid aggregate surface, and by performing further equilibration runs of $1 \mathrm{~ns}$. Thus, water/acid number ratios of 1:1, 2:1, 4:1, and 6:1 have been considered with both carboxylic acid molecules, and, in addition, the number ratio of 10:1 has also been considered in the case of the acetic acid aggregate.

Then, the various acid-water aggregates were progressively heated up to $250 \mathrm{~K}$, increasing the temperature by $10 \mathrm{~K}$ in every 200000 time steps. For five different temperature values (i.e., 150, 180, 200, 220, and $250 \mathrm{~K}$ ), these simulations have been followed by up to $6 \mathrm{~ns}$ long production runs for data analyses. A total number of 55 simulations have thus been conducted to understand the phase behavior of the binary acid-water mixtures. Note that, as in our previous works,[24-26] the temperatures considered here go to much lower values than what is encountered in the troposphere, to shed light on all the possible structural changes in the studied molecular aggregates.

At a first glance, structural characteristics of the water/acid aggregates might simply be determined by looking at equilibrium snapshots. Nevertheless, a more quantitative approach is needed to allow for a detailed analysis of, for instance, hydrogen bonding within the water/acid mixtures. This can be done by calculating size distributions $P(n)$ of the acid and water clusters that may have been formed in the simulations. Criteria for neighboring molecules have thus been determined by considering the first peaks of various radial distribution functions. Two water molecules have been regarded as hydrogen bonded neighbors if the distance between the oxygen atom of one of these molecules and any of the two hydrogen atoms of the other molecule was smaller than $2.50 \AA$. Similarly, two acid molecules have been considered as hydrogen bonded 
neighbors if the distance between an oxygen atom of one of these molecule and the carboxylic or any of the hydrocarbon hydrogen atoms of the other molecule was smaller than $2.25 \AA$, or 3.25 $\AA$, respectively.

In addition, binding energy distributions have been computed in order to get a deeper insight into the energetic background behind structural changes occurring when increasing the temperature or the water mole fraction. Characterizing the interactions within the water/acid mixture can also shed light on the corresponding hydrogen bonding network in the system under study. Thus, the distributions of the binding energy of an acid molecule with all the other acid molecules ( $E_{b}^{\text {acid-acid }}$ ), of a water molecule with all the other water molecules ( $E_{b}^{\text {water-water }}$ ) and of an acid molecule with all the water molecules ( $E_{b}^{\text {acid-water }}$ ) have been calculated. The results are discussed in comparison with our previous studies on dicarboxylic acid-water[24,25] and formic acid-water[26] aggregates, in order to better characterize the possible influence of the $\mathrm{O}: \mathrm{C}$ ratio on the ability of organic aerosols to act as cloud condensation nuclei, as inferred from the analysis of recent experimental results[8].

\section{Results of the simulations}

3.1. Structure of acid aggregates without water. As stated above, simulations of acetic and propionic acid molecules initially scattered randomly in the simulation box resulted in the formation of one single big aggregate irrespective of i) the number of molecules, and ii) the final temperature of the simulations. This aggregate gathers together almost all the acid molecules present in the simulation box, as already observed in previous simulations devoted to formic and malonic acids, $[25,26]$ and in contrast with what was obtained for oxalic acid molecules that were found to form smaller aggregates. This behavior, evidenced by a careful examination of the snapshots issued from the simulations has been confirmed by the analysis of the size distribution $P(n)$ of the clusters formed by the acid molecules in the box. Indeed, only one single peak at high $n$ values was always obtained in $P(n)$ distribution for each system (not shown), corresponding to the formation of a single aggregate containing almost all the acid molecules. Some equilibrium snapshots of the acetic and propionic acid aggregates are shown in Fig. 2 (top) as an illustration.

Note that in the temperature range of the simulations, acetic and propionic acids are in the solid phase and should exhibit a crystalline structure that could have been evidenced by the 
study of some radial distribution functions and by careful examination of the hydrogen bond network in the results of our molecular dynamics simulations. However, the aggregates formed here are characterized by a high ratio of surface to bulk molecules due to their quite small size, preventing thus the formation of well-ordered crystals. Nevertheless, these aggregates showed some local ordering due to hydrogen bonding between acid molecules (see below).

3.2. Phase behavior of the binary aggregates. Acid aggregates have then been placed in a humid environment by adding water molecules to the simulation box with 1:1, 2:1, 4:1, 6:1 (and even 10:1 in the case of acetic acid) water:acid ratios. Simulations have been performed at various temperatures varying between 100 and $250 \mathrm{~K}$. A first analysis of the results has been done simply by looking at selected equilibrium snapshots taken from the simulations, some of them being shown in Fig. 2 as an illustration of the equilibrated systems under investigation. These snapshots clearly show that at low water content (1:1 water:acid ratio), acetic acid molecules (Fig. 2.a) tend to form big aggregates on which surrounding water molecules are adsorbed, irrespective of the temperature. A similar situation has also been observed at higher water contents, but only at low temperatures, typically below $150 \mathrm{~K}$. Indeed, at higher temperature, although the formation of a big molecular aggregate in the simulation box has also been observed, this aggregate is mainly made of water molecules that form its inner core, with very little or no acetic acid molecules (depending on the temperature and the water ratio), whereas the acetic acid molecules are repelled to the surface of the water aggregate. This situation has resulted from the dissolution of the acid aggregate due to the interaction with the water molecules, and to the concomitant migration of the acid molecules to the surface of the water aggregate. At intermediate temperatures, typically between 180 and $200 \mathrm{~K}$, only a partial dissolution of the acid nucleus has been observed, the remaining strongly bound acid molecules are surrounded by a mixed phase of water and acetic acid molecules. A similar situation has been obtained when considering propionic acid aggregates in various humid-like environments, at least for water:acid ratios equal to $1: 1,2: 1,4: 1,6: 1$. As a consequence, we do not simulate the situation corresponding to the largest (10:1) water:acid ratio, which is expected to exhibit the same behavior.

These conclusions have been supported by the analysis of the cluster size distribution functions $P(n)$, which represent the distribution of the number of water or acid molecules that form one aggregate in the simulation box, on the basis of the distance criteria discussed in 
Section 2. It should be noted that in this analysis only neat aggregates of water and acids are considered, i.e., two molecules (either two acids or two waters) are regarded to be part of the same aggregate if they are linked by a chain of neighboring (H-bonded) pairs of the same molecules. Examples of such distributions are shown in Fig. 3 for some acid/water systems, at 150 and $250 \mathrm{~K}$, and for three different water:acid ratios. Note that only a limited number of $P(n)$ distributions are given in Fig. 3 because we choose to not show curves exhibiting similar behavior, for clarity.

At low water content $(1: 1)$ and low temperatures (below $150 \mathrm{~K}), P(n)$ for acetic and propionic acid molecules are characterized by a single peak at the position of $n=120$, indicating that these acid molecules form only one single big aggregate in the simulation box, which is surrounded by the water molecules. Indeed, for these latter molecules, $P(n)$ is characterized by several peaks corresponding to the formation of a limited number of smaller aggregates adsorbed at some parts of the acid surface. Also, some water molecules are even isolated from each other at the surface of the acid aggregate, as indicated by the peak at the $n=1$ value in the $P(n)$ distribution of the water molecules. When increasing the temperature, $P(n)$ of the acid molecules remains basically unchanged, being characterized by a peak at large $n$ values, indicating that acid molecules still form one big aggregate up to $250 \mathrm{~K}$. At the same time, the peaks of the $P(n)$ distribution of water are shifted to lower $n$ values. This finding can be related to a situation when the water molecules, initially aggregated at the surface of the acid grain at low temperature, tend to penetrate into this aggregate due to larger thermal fluctuations when $T$ increases. As a consequence, some water-water and acid-acid pairs at the surface of the aggregate are replaced by water-acid pairs, leading to a decrease of both the water and the acid aggregate sizes.

When the water content is increased, the situation remains similar for the two acid molecules at low temperature, i.e., the corresponding distribution functions $P(n)$ are still characterized by a large peak at around $n=120$. In contrast, the situation is different for water because now the corresponding $P(n)$ distribution usually exhibits only one peak instead of several ones as at the 1:1 water:acid ratio. This finding indicates that all the water molecules are now interconnected within the same network, corresponding to a complete covering of the surface of the acetic acid aggregate by water. The only exception is seen at the water:propionic acid ratio of $4: 1$, two separate peaks are observed in the $P(n)$ distribution of water. This indicates that in this case the propionic acid aggregate is still too large to be fully covered by water 
molecules, and hence water molecules form two relatively large aggregates that are isolated from each other.

At high temperatures, typically above $200 \mathrm{~K}$, although a single peak is still observed in $P(n)$ for the water molecules in both water/acetic and water/propionic acid systems, this peak corresponds to a completely different situation. Indeed, in this case the $P(n)$ distribution of the acid molecules is characterized by a single peak at very small $n$ values, indicating that the acid aggregate stable at low temperature has been fully dissolved in water at higher temperature and at high water content. As a consequence, the single peak observed in $P(n)$ for water cannot be anymore interpreted as a result of the covering of the acid aggregate. Rather, this peak is consistent with the formation of a big water aggregate at the center of the simulation box, in which acid molecules are dissolved at intermediate temperatures (typically between 180 and 220 $\mathrm{K}$ ), or on which they are adsorbed at higher temperatures. This analysis thus fully supports the conclusions already obtained from the snapshot analysis.

To summarize, the first qualitative picture suggested by the analysis of selected snapshots and of the $P(n)$ cluster size distribution functions is that acetic and propionic acid molecules form a single big aggregate at the low 1:1 water:acid ratio, on which water molecules can be adsorbed irrespective of the temperature (demixed phase 1). This situation is still observed at higher water content, but in this case only at low enough temperatures. Indeed, when the temperature is increased, dissolution of the acid aggregate is evidenced by the formation of either a mixed water/acid phase (typically between 180 and $220 \mathrm{~K}$ ), or a demixed phase (above $220 \mathrm{~K}$ ), in which acid molecules are adsorbed at the surface of a big water aggregate (demixed phase 2).

To present these results in a clearer and more compact way, the average cluster sizes of the largest acid aggregates formed in the simulated systems have been calculated. The corresponding results are shown in Fig. 4 as a function of the temperature. First, this figure illustrates that acetic and propionic acid molecules exhibit very similar behavior. In the absence of water, the average size of the acid aggregate remains almost constant, irrespective of the temperature, as already observed simply by looking at the equilibrium snapshots. In contrast, when the acid aggregates are interacting with the water molecules, Fig. 4 clearly evidences two different behaviors corresponding to low and high water content. For a water:acid ratio equal to $1: 1$, only a small decrease of the acid aggregate size is observed upon increase of the temperature, indicating that the acid aggregate does not dissolve into water. This situation is clearly different from what has been obtained when considering the interaction between formic 
acid aggregates and water molecules at the same 1:1 water:acid ratio.[26] Indeed, in this case, a complete deliquescence of the formic acid aggregate was observed above $200 \mathrm{~K}$. Although this different behavior could be a priori related to different water-acid interactions, we have also examined whether or not it depends on the simulation duration, which might have not been not long enough here to observe the whole deliquescence of the acetic and propionic aggregates. However, even a 2 ns increase of the simulation length for the acetic acid / water systems did not significantly modify the previous results, as indicated on Fig. 4. Thus, we can conclude that, at the low water content considered here, acetic and propionic acid aggregates remain stable irrespective of the temperature in the $100-250 \mathrm{~K}$ range.

Upon addition of water, three different temperature regions can be identified on Fig. 4. At low temperatures, typically below $150 \mathrm{~K}$, the average size of the acetic and propionic acid aggregates remains almost constant, irrespective of the water content, indicating the formation of a large acid aggregate in the simulation box, as evidenced by the analysis of Fig. 3. This aggregate is surrounded by water molecules in a situation that corresponds to a pure demixed system. In the high temperature range, typically above $200 \mathrm{~K}$, an almost complete deliquescence of the acid aggregate is obtained, leading to very small values of the average size of the acid clusters. However, it should be emphasized that even at these high temperatures the systems simulated here consist of one large aggregate in the simulation box, as shown by the snapshots given in Fig. 2. However, this aggregate is made of an assembly of water molecules (as evidenced by the $P(n)$ distributions shown in Fig. 3), in which, or at the surface of which, acid molecules are disseminated. For temperatures typically between 150 and $200 \mathrm{~K}$, an intermediate situation has been observed, when the average size of the acid aggregate progressively decreases up to very low values corresponding to the complete deliquescence. However, at a given temperature, a quite surprising and counter-intuitive result has been first obtained regarding the influence of the water:acid ratio. Indeed, the results tend to indicate that the deliquescence of the acid aggregate is more important for a water:acid ratio equal to 4:1 than for higher values. In fact, it turned out that this came from the length of the simulations that has to be increased with the number of water molecules in the box, at temperatures for which thermal motions are still not very large. Much longer simulations have thus been also performed by increasing the simulation runs by 2, 4 and even $6 \mathrm{~ns}$ for each simulation performed at 180, 200 and $220 \mathrm{~K}$. The final results, given in Fig. 4, clearly show the dependence of the average size of the acid aggregate on the simulation length. In fact, it even turned out that a 6 ns-augmented run is still not long 
enough in the case of 10:1 water:acid ratio, leading to the conclusion that the detailed characterization of the intermediate situation of the acid/water systems between $180-200 \mathrm{~K}$ could be a very complex task. Nevertheless, the main conclusion of this work, i.e., stability of the acid aggregate at low, and its complete deliquescence at high temperatures does not depend on the simulation duration. Moreover, it should also be pointed out that increasing the values of the cut-off criteria for determining the cluster size distributions does not changed the present conclusions, as already evidenced in the work devoted to formic acid/water systems.[26]

A more detailed picture of the mixing/demixing behavior of the acid and water molecules can also be obtained from the calculations of the radial distribution functions $g(r)$ of various molecular sites. Here, to characterize the respective locations of the acid and water molecules, four different distributions functions, namely $g_{a c i d}(r), g_{a l k y l}(r), g_{c a r b}(r)$, and $g_{w}(r)$, have been calculated. They are related to the positions of the center of mass of the acid molecule, alkyl chain, carbonyl group, and water molecule,respectively, with respect to the position of the center-of-mass of the big aggregate formed in the simulation box. Examples of these $g(r)$ distribution functions are shown for acetic and propionic acid in Fig. 5 for two temperature values and four water-acid ratios. At low temperatures (typically up to $180 \mathrm{~K}), g_{\text {acid }}(r)$ is characterized by several peaks indicating the formation of a more or less structured acid aggregate, at the surface of which water molecules are adsorbed, as indicated by the position of the main peak of $g_{w}(r)$, irrespective of the water:acid ratio. In contrast, at higher temperatures, $g_{w}(r)$ is typical of the formation of a water droplet, at the surface of which the acid molecules are adsorbed, with the exception of the system containing a small number of water molecules (1:1 water:acid ratio), in which the acid aggregate is still existing in the simulation box. In this case, however, $g_{w}(r)$ clearly indicates that water and acid molecules are mixed within the surface layer of the acid aggregate.

In addition, the analysis of $g_{\text {alkyl }}(r)$ and $g_{\text {carb }}(r)$ shows that the carboxylic group of the acid molecules is always located closer to water molecules than the alkyl part of the acids, indicating that the acid molecules are oriented with their hydrophilic head toward waters, as expected. This information is of particular importance when considering the systems simulated at high water content and temperatures above $200 \mathrm{~K}$, i.e., systems for which the acid molecules are preferentially adsorbed at the surface of the water aggregate. Indeed, $g_{\text {alkyl }}(r)$ indicates that, at the interface between the resulting acid/water aggregate and the gas phase, acid molecules orient 
their hydrophobic group toward the gas phase, a behavior that could influence the propensity of organic aerosols to act as cloud condensation nuclei.

3.3. Energetic background. The energetic reasons underlying the behavior of the acid/water systems studied above can be characterized by the analysis of the binding energy distributions for the acid-acid ( $\left.E_{b}^{\text {acid-acid }}\right)$, acid-water $\left(E_{b}^{\text {acid-water }}\right)$ and water-water ( $\left.E_{b}^{\text {water-water }}\right)$ pairs. Examples of such curves are given in Figs. 6 a and $b$ for acetic and propionic acid at two different temperatures and for three water ratios. Indeed, the overall behavior of these curves is quite similar for the two acid molecules considered here.

At low temperatures (up to $150 \mathrm{~K}$, first columns of Fig. 6), the acid-acid, acid-water and waterwater binding energy distributions do not really depend on the water content in the simulated systems. Both $E_{b}^{\text {water-water }}$ and $E_{b}^{\text {acid-acid }}$ binding energies are characterized by a very broad distribution. Thus, peaks around $-50,-75$ and $-100 \mathrm{~kJ} / \mathrm{mol}$ can be seen for the water-water binding energy. Assuming that the average energy of a single hydrogen bond is around -25 $\mathrm{kJ} / \mathrm{mol}$ for the TIP5P water potential used here [38], this indicates that the water molecules usually form between two and four hydrogen bonds with their water neighbors. In the same way, the broad peak observed in the $E_{b}^{\text {acid-acid }}$ distribution can be related to the formation of several hydrogen bonds between acid molecules. The energy distribution of the acid-water interactions presents, in turn, two broad peaks, the first one being located around $-80 \mathrm{~kJ} / \mathrm{mol}$ and the second one between -20 and $0 \mathrm{~kJ} / \mathrm{mol}$. Whereas the former peak corresponds to acid molecules that strongly interact with the water molecules located in their neighborhood, forming typically three hydrogen bonds with them, the latter peak indicates that there are also several acid molecules that are too far from the water molecules to interact strongly with them. At these low temperatures, the picture that thus emerges from this analysis is that the energy distributions are consistent with the formation of a large acid aggregate at the surface of which water molecules are agglomerated, irrespective of the water content.

At high temperature $\left(250 \mathrm{~K}\right.$, second columns of Fig. 6), the distribution of $E_{\mathrm{b}}^{\text {acid-acid }}$ shifts toward higher (i.e., less negative) energy values, showing that the acid molecules tend to lose their hydrogen bonded acid neighbors. Note however, that the corresponding peak is much broader at low than at high water content, indicating that some strong acid-acid interactions remain in the former case. At the same time, the interaction between acid and water molecules 
increases, as indicated by the disappearance of the high energy peak (i.e., the one between -20 and $0 \mathrm{~kJ} / \mathrm{mol}$ ) in the acid-water binding energy distribution. These two features clearly evidence the increase of the interaction between acid and water molecules at the expense of the interactions between acid molecules. However, the most interesting information comes from the water-water binding energy distribution, which strongly depends on the water content in the $\mathrm{acid} /$ water systems considered here. Indeed, the distribution of $E_{b}^{\text {water-water }}$ is characterized by a single broad peak located at around $-25 \mathrm{~kJ} / \mathrm{mol}$ at low water content (1:1 water:acid ratio), and around $-75 \mathrm{~kJ} / \mathrm{mol}$ at higher water contents (4:1 and $6: 1$ in the case of propionic acid). At these high water contents, the water-water binding energy distribution is thus consistent with the formation of a big water aggregate in the simulation box, at the surface of which acid molecules are adsorbed. In contrast, at low water content, the peak at $-25 \mathrm{~kJ} / \mathrm{mol}$ indicates a much weaker water-water binding and, it is consistent with water molecules located quite far from each other, i.e., scattered at the surface layer of the acid aggregate, the persistence of which being indicated by the long tail at the low energy side of the peak of the $E_{b}^{\text {acid-acid }}$ binding energy distribution.

Summarizing, the analysis of the energy distributions in the acid/water systems considered here supports the existence of a transition from one demixed to another demixed phase for the binary acid+water systems upon temperature increase at high water content. The first situation corresponds to the demixed phase 1, in which water molecules are adsorbed on the acid aggregate, whereas the second situation corresponds to the demixed phase 2 , in which the acid molecules are adsorbed at the surface of the water aggregate. At intermediate temperatures, a mixture between acid and water molecules is obtained, in particular, within the surface layer of the mixed aggregate. In contrast, at low water content, acid and water remains in the demixed phase 1 in the entire temperature range considered, as already suggested by the analyses of the snapshots, the cluster size distributions and the radial distribution functions.

\section{Discussion}

The present results have evidenced a very similar behavior between acetic and propionic acid aggregates when surrounding by water molecules, irrespective of the temperature. The comparison with our previous results obtained when characterizing, with the same modeling approach, the behavior of binary systems made of water and formic acid, [26] i.e., the smallest monocarboxylic acid molecule, also shows a similar behavior, but only at low temperature. 
Indeed, for formic acid, two different phases have been evidenced, corresponding either to water adsorption on formic acid aggregates at low temperature (demixed phase) or to the formation of mixed droplets consisting of formic acid and water molecules at higher temperatures (mixed phase), irrespective of the water contents considered in the corresponding simulations. Here, although we evidenced adsorption of water around acetic and propionic acid aggregates at low temperature (demixed phase 1), similarly to the formic acid/water systems, different situations have been seen at higher temperatures, depending on the water content. Indeed, at low water content, a large part of the acetic and propionic acid molecules remain aggregated even at high temperature, the mixing with water being observed only within the surface layer of the acid aggregate. This feature can be related to the acid-acid interactions within the acid aggregate, which is found to be slightly stronger in the simulations of acetic and propionic acid than in those of formic acid. Moreover, at high water content and high temperature, acetic and propionic acid molecules are clearly repelled to the surface of the water aggregate that is formed in the simulation box (demixed phase 2). This "inverted micelle-like" behavior can be clearly attributed to the hydrophobic part of the acetic and propionic acid molecules (i.e., to the alkyl chain), and may explain why it has not been observed in the simulations performed with formic acid. It should be noted that, because of the results obtained here on acetic and propionic acids, we have performed additional calculations on the formic acid/water systems with simulation times considerably longer than in our previous study [26] to investigate the effect of a possible artifact coming from too short simulation time. However, it turns out than even after $20 \mathrm{~ns}$ of simulations at $250 \mathrm{~K}$, about $15 \%$ of the formic acid molecules are still mixed in the water aggregate, the other formic acid molecules being adsorbed at its surface. It thus appears that, when interacting with water, acetic and propionic acid molecules tend to be much more easily demixed than formic acid molecules. Such a behavior has also been observed for oxalic acid molecules, which have been shown to form demixed droplets at high temperature and high water contents [24], contrarily to malonic acid molecules.[25]

Of course, it should be reminded that the simulation results depend on the model used to describe the interactions between the different species in the simulation box, i.e., not only the water-water and acid-acid, but also the acid-water interactions. Although the accuracy of the former one have been widely investigated in the literature (see for instance Vega et al.[40]), the validity of the acid-acid and, even worse, of the acid-water interactions has not been widely studied. For instance, the OPLS parameters used for the acid molecules have been originally 
developed for acetic acid molecules only, [34] but the corresponding parameters have been usually used to describe other acid-acid interactions, until these parameters have been modified to account for a more general description of acid molecules.[35] However, Jedlovszky and Turi showed that this parameterization may be not sufficiently accurate and, as a consequence, they have proposed a new potential model for formic acid in liquid simulations.[41] Similarly, Kamath et al. developed an improved force field for the prediction of the vapor-liquid equilibria for carboxylic acids, with a special focus on pentanoic and octanoic acids.[42] Unfortunately, we are not aware of such tests for propionic acid, and hence we have used here the standard OPLS parameter values. Moreover, to the best of our knowledge, there are no available studies specifically devoted to testing the accuracy of the interaction between water and acetic or propionic acid molecules.

From the experimental point of view, to the best of our knowledge, there is only one experimental study that has been devoted to the structural characterization of acetic acid-ice aerosol particles by means of infrared spectroscopy measurements. Although these experiments were conducted at $78 \mathrm{~K}$ and with much bigger aerosol particles (10 to $600 \mathrm{~nm}$ ) than what we have simulated, we can make a tentative comparison between the experimental findings and ours, at least qualitatively. The experimental results showed that pure acetic acid aerosols tend to exhibit a partial crystal form, which agrees quite well with our findings. Then, at 1:1 water:acid ratio, no mixture on a molecular level has been evidenced between acetic and water molecules from the analysis of the infrared spectra, leading to the conclusion that these two species form a two-phase mixture, which can be related to a demixed phase (demixed phase 1, obtained in the simulations at low temperature). At high water content (10:1 water:acid ratio) the experimental results suggested the existence of an amorphous mixture of acid and water, which, again, agrees quite well with the mixing phase evidenced in the surface layer of the aggregates simulated here at high water content but at higher temperatures.

It is also interesting to discuss the ability of the aggregates simulated here to act as nuclei for further water condensation at tropospheric temperatures. At these temperatures, our results show that acetic and propionic acid form stable aggregates on which water molecules can be adsorbed. However, when the number of water molecules increases (i.e., when increasing the water:acid ratio), the dissolution of the acid aggregate is obtained with the concomitant aggregation of the water molecules and the migration of the acid molecules to the surface of the resulting water aggregate. The surface of the water/acid systems is thus made of a mixture of 
water and acid molecules, the latter ones being adsorbed at the surface of the water aggregate and oriented in such a way that they have their hydrophobic tail directed toward the gas phase. The subsequent growth of the particle could thus depend on the surface ratio of the water and acid molecules, i.e., on the probability that an incoming water molecule will interact with a surface water (strongly attractive interaction) or with a surface acid molecule (weak interaction).

In a recent study it has been proposed, on the basis of water adsorption experiments on acid films in a Knudsen cell flow reactor, that the O:C ratio could be used as a proxy for determining the heterogeneous ice nucleation efficacy of organic acid aerosols.[8] However, it is worth noting that, after compiling all the conclusions issued from simulations of different water/acid systems (including formic,[26] acetic, propionic, oxalic,[24] and malonic [25] acids), it is clear that the use of the $\mathrm{O}: \mathrm{C}$ ratio as a single criterion is not sufficient to infer the propensity of organic acid aerosols to act as cloud condensation nuclei. Instead, our conclusions show that this behavior depends on several factors, such as the number of the acidic groups (i.e., mono- vs. bifunctional acids), or the size of the molecule, and the O:C ratio is only one, although important, among these factors.

Finally, it is worth noting that the "inverted micelle" like behavior, evidenced here at

temperatures and compositions relevant to the Troposphere both for acetic and propionic acid molecules in contact with water grains, agrees quite well with some usual models of atmospheric aerosols that consists of an aqueous core encapsulated in an inert, hydrophobic organic layer [43].

\section{Summary and Conclusions}

Water adsorption around small acetic and propionic acid aggregates has been studied by means of molecular dynamics simulations in the temperature range of 100-250 K, including thus temperatures relevant for the Troposphere. Systems corresponding to various water contents have been considered, allowing us to characterize the different phases of the binary acid-water systems. Calculations have shown that acetic and propionic acid molecules behave similarly, and both the temperature and the water content have a strong influence on the behavior of the systems.

Two situations have been evidenced for the acid-water aggregates, corresponding either to water adsorption on large acid grains at very low temperatures (demixed phase 1), or to the formation of demixed droplets consisting of acid molecules adsorbed at the surface of 
water aggregates at higher temperatures and high water content (demixed phase 2). At low water content and high temperature, only a partial mixing between water and acid molecules is observed, in particular, at the surface of the aggregate. At moderate temperatures, an intermediate situation is obtained, which characterized by a partial deliquescence of the acid aggregate (mixed phase).

In addition, the comparison between the present results and those obtained previously for formic, [26] oxalic [24] and malonic acids [25] reinforces our previous conclusions that using the $\mathrm{O}: \mathrm{C}$ ratio for predicting the ability of carboxylic acids to act as water nuclei might be unfortunately not as straightforward as previously inferred.[8] At least, it seems that the affinity of these acid molecules for water depends not only on the O:C ratio, but also on their number of carboxylic groups and of their internal geometry.

Of course, the present results cannot be directly compared to any field measurements. However, they lead to a deeper understanding of the complicated and environmentally relevant problem of heterogeneous nucleation of water, and can thus be viewed as an additional step towards modeling of organic cloud condensation nuclei. Above all, the results of the present simulations emphasize the need for further experimental and simulation works for a better characterization of the complex effect of both temperature and humidity on the behavior of organic aerosols.

Acknowledgements. This project is supported by the Observatoire OSU THETA of Besançon, by the Hungarian OTKA Foundation under project No. 104234, and by the European Union, co-financed by the European Social Fund in the framework of the Social Renewal Operative Program under the project name "Research, Innovation, Collaborations - Social innovation and strenghtening of research collaboration networks in collaboration with Eszterházy Károly College, Bay Zoltán Applied Research Nonprofit Ltd. and Agria TISZK Nonprofit Public Benefit Ltd" and project number TÁMOP 4.2.1.D-15/1/KONV-2015-0013. Simulations have been executed on Institut UTINAM's computers supported by the Région de Franche-Comté and the CNRS/INSU and also using the computing resources of the Mésocentre de Calcul, a regional computing center at Université de Franche-Comté. 


\section{References}

(1) Andreae, M.O.; Gelencser, A. Atmos. Chem. Phys. 2006, 6, 3131-3148.

(2) Lazaridis, M. 2008, in Environmental chemistry of aerosols, (Editor I. Colbeck; Blackwell Publishing, Oxford), p.93

(3) Kanakidou, M.; Seinfeld, J.H.; Pandis, S.N.; Barnes, I.; Dentener, F.J.; Facchini, M.C.; Van Dingenen, R.; Ervens, B.; Nenes, A.; Nielsen, C.J.; Swietlicki, E.; Putaud, J.P.. Balkanski, Y.; Fuzzi, S.; Horth, J.; Moortgat, G.K.; Winterhalter, R.; Myhre, C.E.L.; Tsigaridis, K.; Vignati, E.; Stephanou, E.G.; Wilson, J. Atmos. Chem. Phys. 2005, 5, 1053-1123.

(4) Satheesh, S.K.;Krishna Moorthy, K. Atmos. Environ. 2005, 39, 2089-2110.

(5) Lohmann, U.; Feichter, J. Atmos. Chem. Phys. 2005, 5, 715-737.

(6) Seinfeld, J.H.; Pandis, S.N. 1998. Atmospheric chemistry and physics. From air pollution to climate change. Wiley, New York.

(7) Sun, J.; Ariya, P.A. Atmos. Environ. 2006, 40, 795-820 and references therein.

(8) Schill, G.P.; Tolbert, M.A. J. Phys. Chem. A 2012, 116, 6817-6822.

(9) Romanias, M.N.; Papadimitriou, V.C.; Papagiannakopoulos, P. J. Phys. Chem. A 2014, $118,11380-11387$.

(10) Picaud, S.; Hoang, P.N.M. J. Chem. Phys. 2000, 112, 9898-9908.

(11) Collignon, B.; Picaud, S. Chem. Phys. Lett. 2004, 393, 457-463.

(12) Peybernès, N. ; Le Calvé, S. ; Mirabel, Ph.; Picaud, S. ; Hoang, P.N.M. J. Phys. Chem. B 2004, 108, 17425-17432.

(13) Picaud, S.; Hoang, P.N.M.; Peybernès, N.; Le Calvé, S.; Mirabel, Ph.; J. Chem. Phys. 2005, 122, 194707.

(14) Jedlovszky, P.; Partay, L.B.; Hoang, P.N.M.; Picaud, S.; van Hessberg, Ph.; Crowley, J.N. J. Am. Chem. Soc. 2006, 128, 15300-15308.

(15) Hantal, G.; Jedlovszky, P.; Hoang, P.N.M.; Picaud, S. J. Phys. Chem. C 2007, 111, 14170-14178.

(16) Hantal, G.; Jedlovszky, P.; Hoang, P.N.M.; Picaud, S. Phys. Chem. Chem. Phys. 2008, 10, 6369-6380.

(17) Jedlovszky, P.; Hantal, G.; Neurohr, K.; Picaud, S.; Hoang, P.N.M.; von Hessberg, Ph.; Crowley, J.N. J. Phys. Chem. C 2008, 112, 8976-8987. 
(18) Darvas, M.; Picaud, S.; Jedlovszky, P. ChemPhysChem 2010, 11, 3971-3979.

(19) Petitjean, M.; Darvas, M.; Le Calvé, S.; Jedlovszky, P.; Picaud, S. ChemPhysChem 2010, 11, 3921-3927.

(20) Darvas, M.; Lasne, J.; Laffon, C.; Parent, Ph.; Picaud, S.; Jedlovszky, P. Langmuir 2012, 28, 4198-4207.

(21) Mészár, Z.E.; Hantal, G.; Picaud, S.; Jedlovszky, P. J. Phys. Chem. C 2013, 117, 6719-6729.

(22) Picaud, S.; Jedlovszky, P. Chem. Phys. Lett. 600, 73-78 (2014).

(23) Sumi, I.; Picaud, S.; Jedlovszky, P. J. Phys. Chem. C (2015) sous presse

(24) Darvas, M.; Picaud, S.; Jedlovszky, P. Phys. Chem. Chem. Phys. 2011, 13, 1983019839.

(25) Darvas, M.; Picaud, S.; Jedlovszky, P. Phys. Chem. Chem. Phys. 2013, 15, 1094210951.

(26) Vardanega, D.; Picaud, S. J. Chem. Phys. 2014, 141, 104701.

(27) Li, X. ; Hede, T. ; Tu, Y. ; Leck, C. ; Ågren, H. J. Phys. Chem. Lett. 2010, 1, 769-773.

(28) Hede, T. ; Li, X. ; Leck, C.; Tu, Y. ; Ågren, H. Atmos. Chem. Phys. 2011, 11, 65496557.

(29) Ma, X.; Chakraborty, P. ; Henz, B.J. ; Zachariah, M.R. Phys. Chem. Chem. Phys. 2011, 13, 9374-9384.

(30) Sun, L. ; Li, X. ; Hede, T. ; Tu,Y. ; Leck, C. ; Ågren, H. J. Phys. Chem. B 2012, 116, 3198-3204.

(31) Li, X. ; Hede, T. ; Tu, Y. ; Leck, C. ; Ågren, H. Tellus B 2013, 65, 20476.

(32) Sun, L. ; Hede, T. ; Tu, Y. ; Leck, C. ; Ågren, H. J. Phys. Chem. A 2013, 117, 1074610752.

(33) van der Spoel, D; Lindahl, E.; Hess, B.; van Buuren, A. R.; Apol, E.; Meulenhoff, P. J.; Tieleman, D. P. ; Sijbers, A. L. T. M.; Feenstra, K. A. ; van Drunen, R.; Berendsen, H. J. C. GROMACS User Manual version 4.5.4, www.gromacs.org (2010)

(34) Briggs, J. M.; Nguyen, T. B.; Jorgensen, W. L. J. Phys. Chem. 1991, 95, 3315-3322.

(35) Jorgensen, W. L.; Maxwell, D.S.; Tirado-Rives, J. J. Am. Chem. Soc. 1996, 118, $11225-11236$ 
(36) Malde, A.K.; Zuo, L.; Breeze, M.; Stroet, M.; Poger, D.; Nair, P.C.; Oostenbrink, C.; Mark, A.E. J. Chem. Theory Comput. 2011, 7, 4026-4037.

(37) Canzar, S.; El-Kebir, M.; Pool, R.; Elbassioni, K.; Malde, A.K.; Mark, A.E.; Geerke, D.P.; Stougie, L.; Klau, G.W. J. Comput. Bio. 2013, 20, 188-198.

(38) Koziara, K.B.; Stroet, M.; Malde, A.K.; Mark, A.E. J. Comput. Aided Mol. Des. 2014, 28, 221-233.

(39) Mahoney, M.W.; Jorgensen, W.L. J. Chem. Phys. 2000, 112, 8910-8922.

(40) Vega, C.; Abascal, J.L.F.; Conde, M.M.; Aragones J.L. Faraday Discuss. 2008, 141, $1-26$.

(41) Jedlovszky, P.; Turi, L. J. Phys. Chem. A 1997, 101, 2662-2665; Corrigendum J. Phys. Chem. A 1999, 103, 3796.

(42) Kamath, G.; Cao, F.; Potoff, J.J. J. Phys. Chem. B 2004, 108, 14130-14136

(43) Ellison, G.B.; Tuck, A.F.; Vaida, V. J. Geophys. Res. 1999, 104, 11633-11641. 


\section{Tables}

Table 1. Lennard-Jones parameters ( $\sigma$ in $\AA$ and $\varepsilon$ in $\mathrm{kJ} / \mathrm{mol}$ ) and fractional charges (q/e) located on the different atoms of the acetic [34], propionic [35] and water molecules [39] in the potential models used.

\begin{tabular}{llllllllllll}
\hline \hline \multicolumn{2}{c}{ Acetic acid } & \multicolumn{1}{c}{ Propionic acid } & \multicolumn{7}{c}{ Water } \\
\hline atom & $q / \mathrm{e}$ & $\sigma$ & $\varepsilon$ & atom & $q / \mathrm{e}$ & $\sigma$ & $\varepsilon$ & atom & $q / \mathrm{e}$ & $\sigma$ & $\varepsilon$ \\
\hline $\mathrm{C}(\mathrm{C}=\mathrm{O})$ & 0.55 & 3.75 & 0.439 & $\mathrm{C}(\mathrm{C}=\mathrm{O})$ & 0.52 & 3.75 & 0.439 & $\mathrm{O}$ & 0 & 3.12 & 0.669 \\
$\mathrm{O}(\mathrm{O}=\mathrm{C})$ & -0.5 & 2.96 & 0.879 & $\mathrm{O}(\mathrm{O}=\mathrm{C})$ & -0.44 & 2.96 & 0.879 & $\mathrm{H} 1$ & 0.241 & 0 & 0 \\
$\mathrm{O}(\mathrm{O}-\mathrm{H})$ & -0.58 & 3.07 & 0.711 & $\mathrm{O}(\mathrm{O}-\mathrm{H})$ & -0.53 & 3.00 & 0.711 & $\mathrm{H} 2$ & 0.241 & 0 & 0 \\
$\mathrm{H}(\mathrm{H}-\mathrm{O})$ & 0.45 & 0 & 0 & $\mathrm{H}(\mathrm{H}-\mathrm{O})$ & 0.45 & 0 & 0 & $\mathrm{M} 1$ & -0.241 & 0 & 0 \\
$\mathrm{C}\left(\mathrm{CH}_{3}\right)$ & 0.08 & 3.91 & 0.669 & $\mathrm{C}\left(\mathrm{CH}_{2}\right)$ & -0.12 & 3.50 & 0.276 & $\mathrm{M} 2$ & -0.241 & 0 & 0 \\
$\mathrm{H} 1$ & 0 & 0 & 0 & $\mathrm{H} 1$ & 0 & 0 & 0 & & & & \\
$\mathrm{H} 2$ & 0 & 0 & 0 & $\mathrm{H} 2$ & 0 & 0 & 0 & & & & \\
$\mathrm{H} 3$ & 0 & 0 & 0 & $\mathrm{C}\left(\mathrm{CH}_{3}\right)$ & -0.18 & 3.50 & 0.276 & & & & \\
& & & & $\mathrm{H} 3$ & 0 & 0 & 0 & & & & \\
& & & & $\mathrm{H} 4$ & 0 & 0 & 0 & & & \\
\end{tabular}




\section{Figure captions}

Figure 1. Molecule models for acetic, propionic and water molecules used in the simulations. $\mathrm{O}, \mathrm{C}$ and $\mathrm{H}$ atoms are represented by red, light blue, and white balls. Note that $\mathrm{M}_{1}$ and $\mathrm{M}_{2}$ (represented by rose balls) are two additional sites used in the TIP5P model of water to better represent the charge distribution of the water molecule.[38]

Figure 2. Equilibrium snapshots of (a) acetic and (b) propionic acid aggregates at 150, 200 and $250 \mathrm{~K}$ (left, middle, and right) and (from top to bottom) for 0, 1:1, 4:1, 6:1 water:acid ratios (the results for additional simulations at 10:1 water:acid ratio are also shown for acetic acid). For the acid molecules, $\mathrm{O}, \mathrm{C}$ and $\mathrm{H}$ atoms are represented by red, light blue, and white balls whereas all the atoms of water molecules are represented in dark blue for clarity.

Figure 3. Cluster size distributions $P(n)$ of (a) acetic and (b) propionic acid (black curves) and water (red curves) molecules in the acid-water binary aggregates for different water contents, at 150 and $250 \mathrm{~K}$. A value $P(n)=1$ for size $n$ means that all the corresponding molecules (acid or water) form an aggregate of size $n$ in the simulation box.

Figure 4. Average cluster size of (a) acetic and (b) propionic acid aggregates formed in the acid-water systems corresponding to various water:acid ratios at temperatures ranging from 100 to $250 \mathrm{~K}$. For each system, results from simulations of increasing duration length are indicated (see text).

Figure 5. Radial distribution functions $g(r)$ of water (dark blue) and (a) acetic and (b) propionic acid (black) molecules with respect to the aggregate center of mass for different water contents in the simulation box and two different temperatures. Red and green curves represent the radial distribution function calculated separately for the hydrophobic tail and and the hydrophilic head of the acid molecules, respectively.

Figure 6. Binding energy distributions of (a) acetic and (b) propionic acid aggregates interacting with different amounts of water molecules in the simulation box, at 150 and 250 
K. Black and blue curves represent the binding energy of an acid molecule with all the other acid molecules, and of a water molecule with all the other waters in the system, respectively. The red curves represent the binding energy between an acid molecule and all the water molecules in the simulation box. 
Figure 1.

Radola et $a l$.

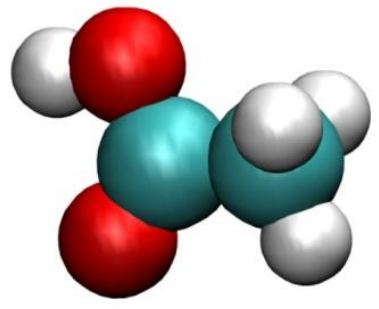

acetic acid

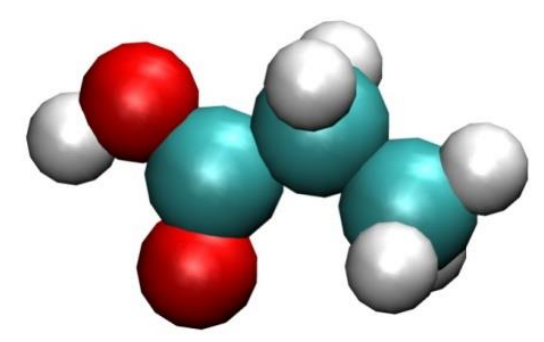

propionic acid

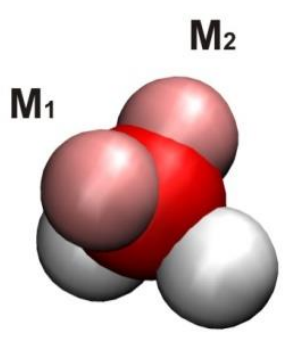

TIP5P water 


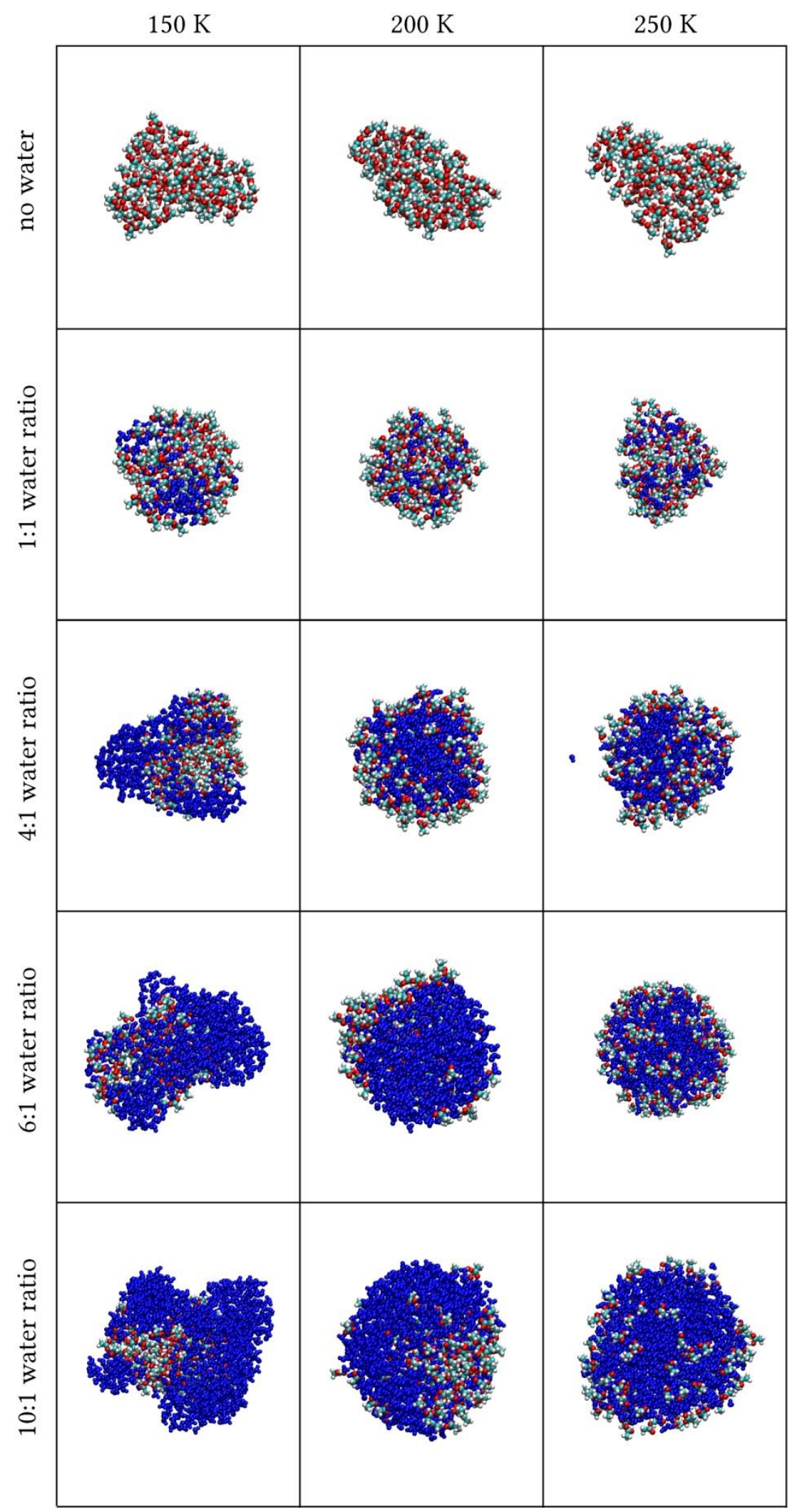

Figure 2.a.

Radola et $a l$. 
Figure 2.b.

Radola et $\boldsymbol{a l}$.

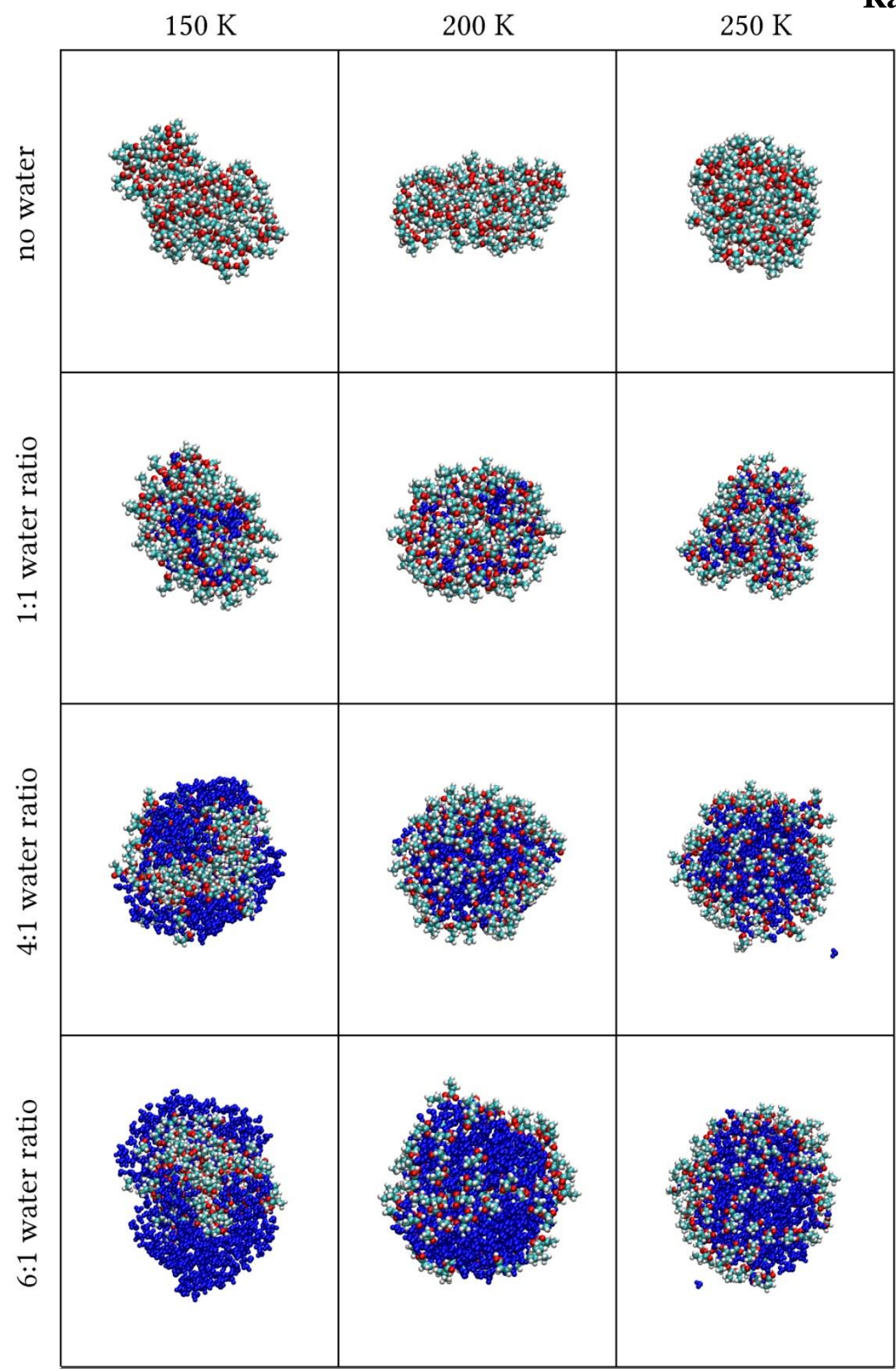


Figure 3.

Radola et $a l$.
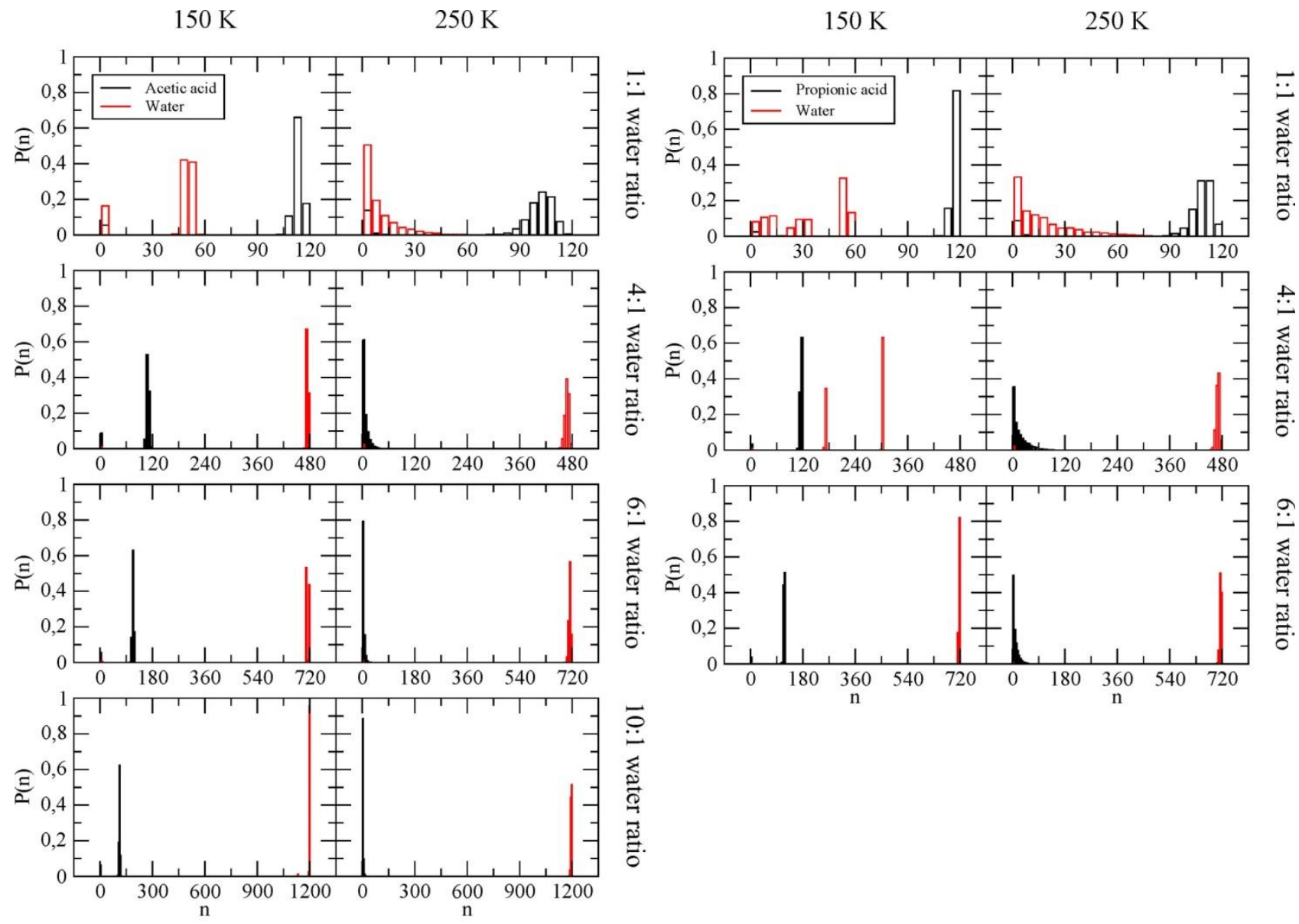

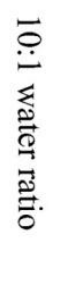

(a)

(b) 
Figure 4.

Radola et $a l$.

(a)

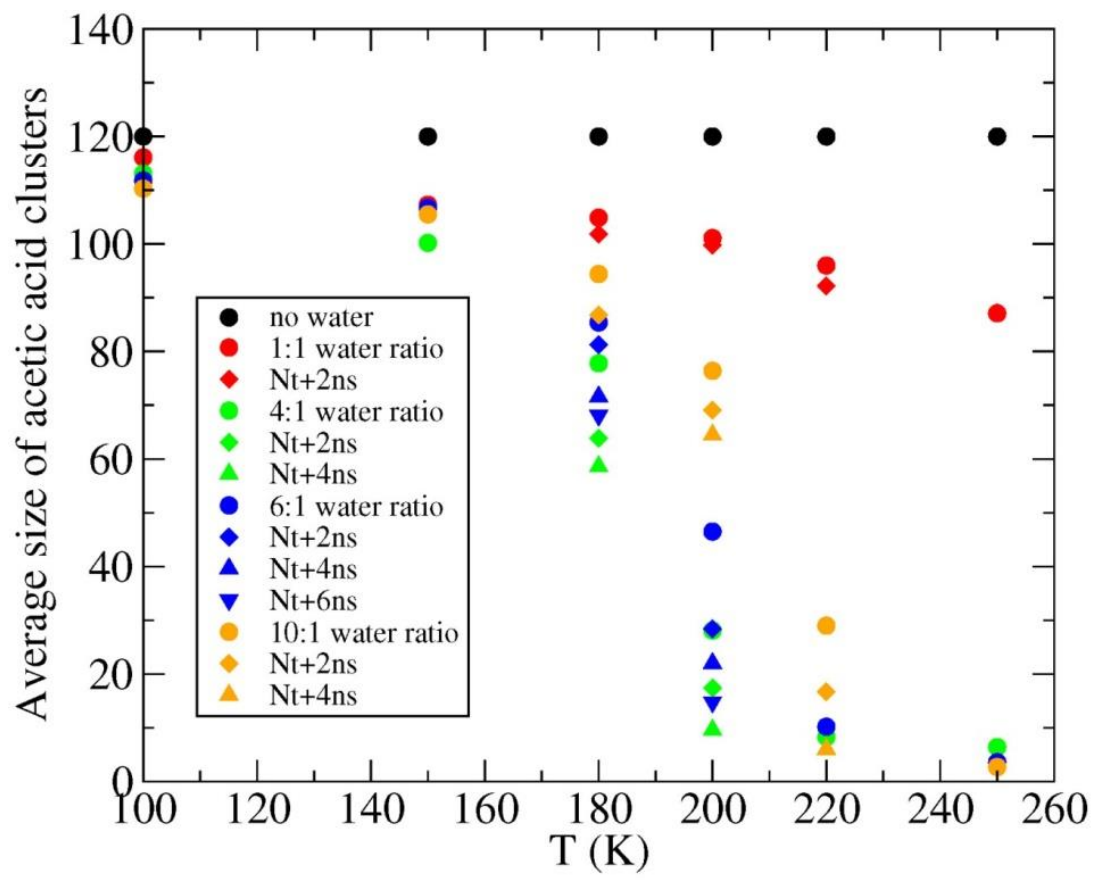

(b)

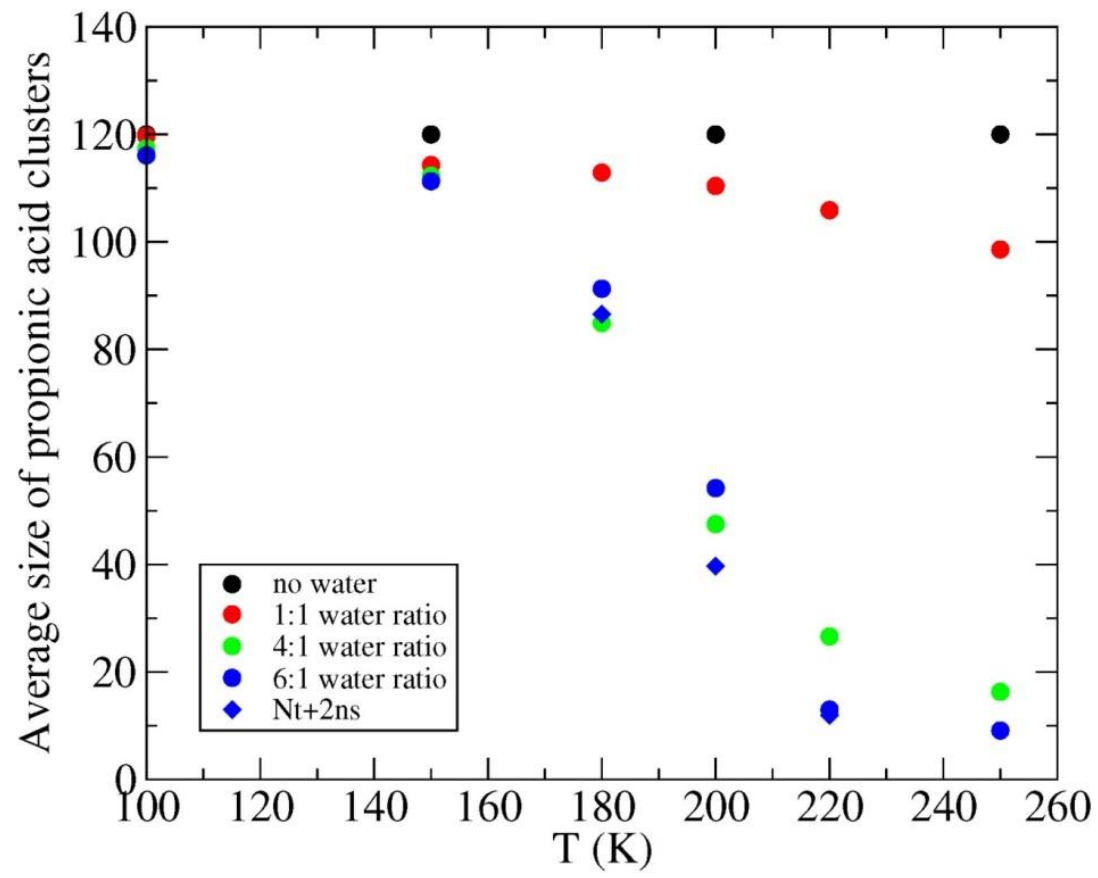


Figure 5.

Radola et $a l$.

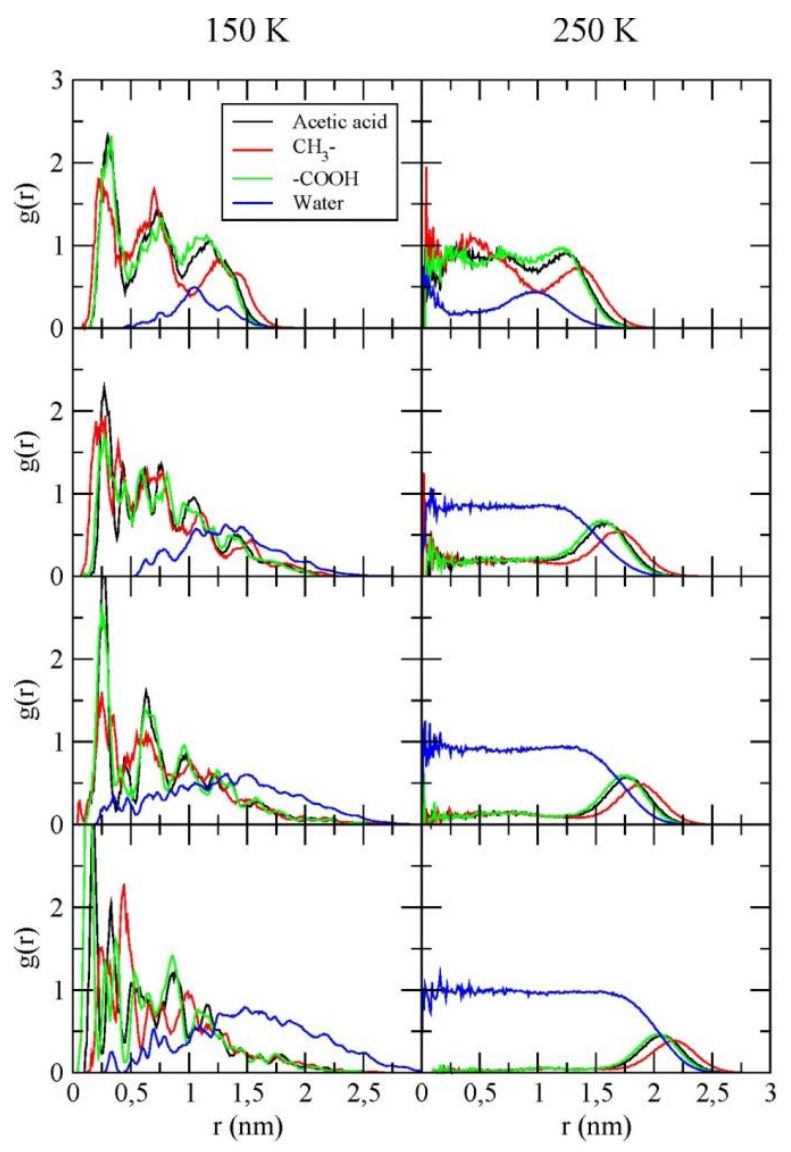

(a)

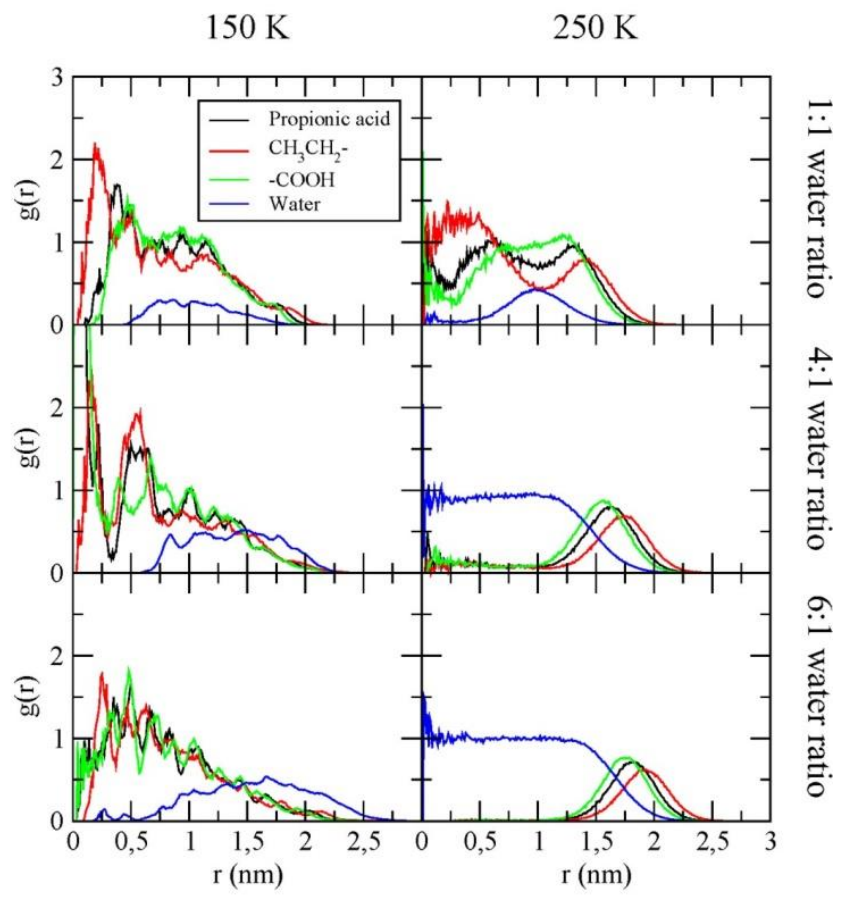

(b) 
Figure 6.

Radola et $a l$.

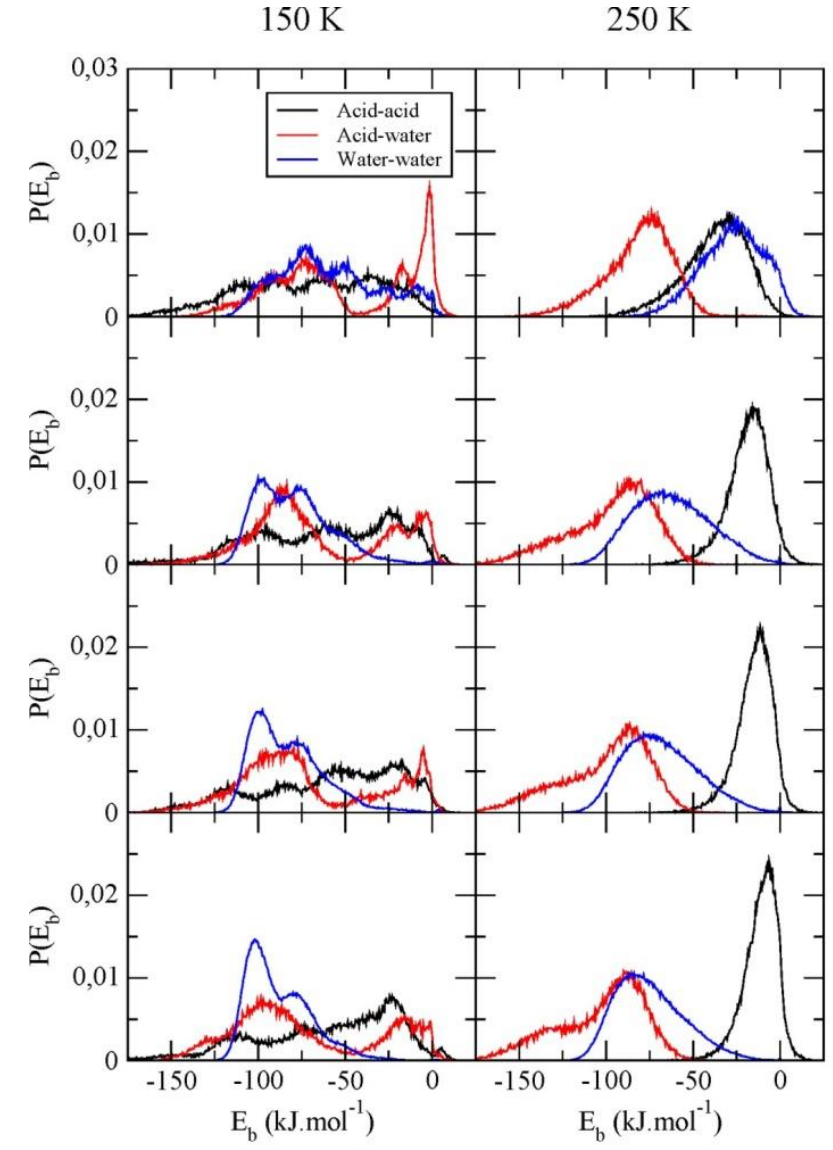

(a)

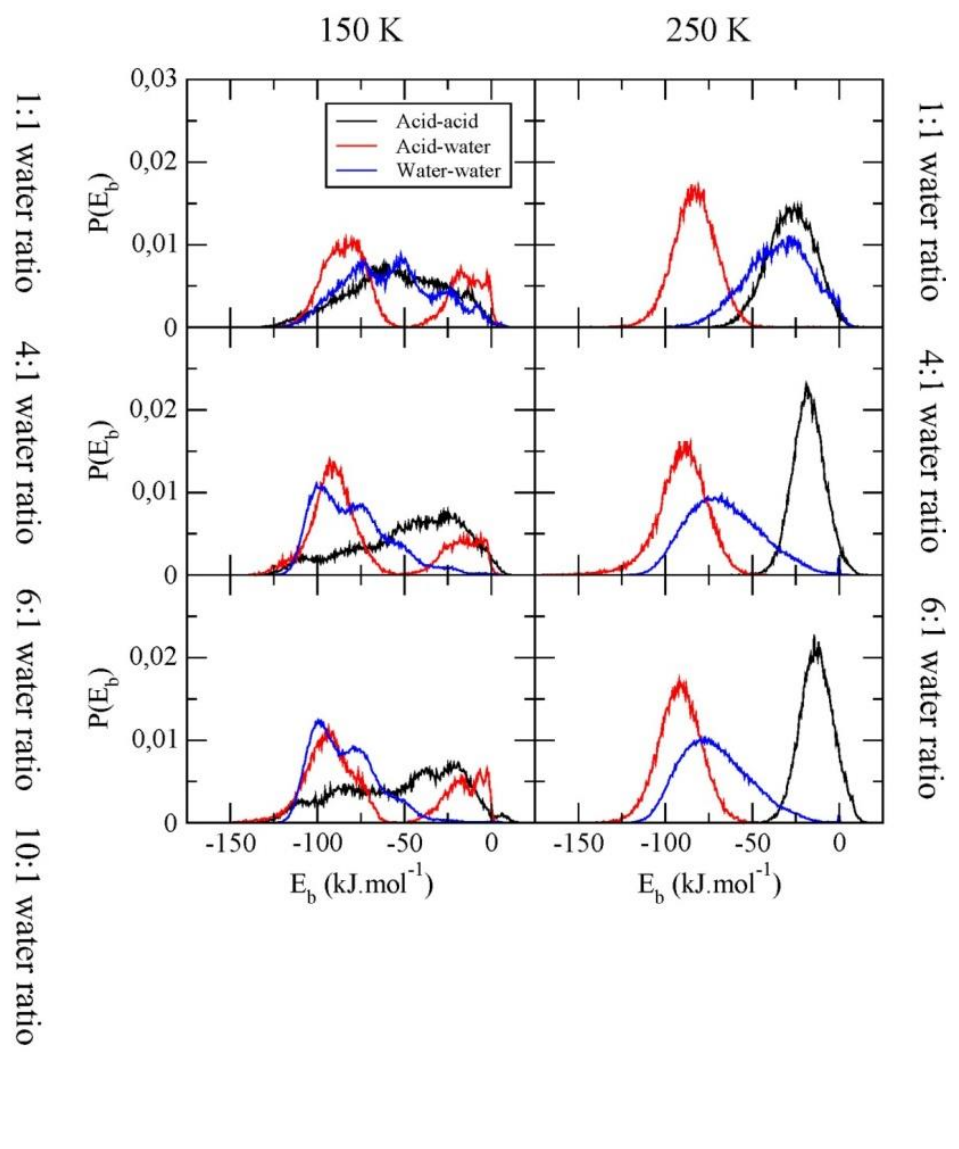

(b) 